\title{
Articles
}

\author{
MARIA MEDINA-VICENT ${ }^{1}$ \\ SONIA REVERTER-BAÑÓN²
}

\section{La perspectiva de género como una mirada crítica al mundo ${ }^{3}$}

\section{The Gender Perspective as a Critical Look to the World}

La perspectiva de género puede ser aplicada a cualquier campo de conocimiento y actividad humana, ya que en todos estos espacios de conocimiento e interacción las relaciones entre mujeres y hombres son la clave central de funcionamiento. Teniendo en cuenta que los patrones asimétricos de poder se reproducen en todas las dimensiones de lo social, y que la injusticia generada por estos debe ser enfrentada de forma transversal, la aplicación de la perspectiva de género se vuelve necesaria y urgente en cualquier ámbito de la vida social al que nos queramos remitir. Este número es un ejemplo claro de que dicha perspectiva se fundamenta en una mirada siempre heterogénea y diversa (López Saavedra, 2011), además de interseccional (Crenshaw, 1989), que se aproxima críticamente a la realidad del mundo con el objetivo principal de transformar cualquier realidad injusta.

«Miradas feministas que interrogan» es el número veintinueve de la revista Asparkía. Investigació Feminista, y en su seno se aglutinan reflexiones sobre la cuestión de género en diferentes campos de estudio que nos permiten reflexionar al mismo tiempo sobre la transversalidad de la perspectiva de género (Verloo, 2005). Como el/la lector/a podrá comprobar a lo largo de este viaje, la heterogeneidad de temáticas y aproximaciones realizadas por los/as autores/as nos hace ser testigos de que la lucha feminista es multidimensional y se encuentra activa en diferentes frentes.

Así, a lo largo de «Miradas feministas que interrogan» podemos encontrar aproximaciones a la perspectiva de género en diferentes ámbitos de investigación como la literatura, la antropología, el arte, la estética y la filosofía; realidad que nos traslada a su vez la necesidad de «mantener un equilibrio constante entre las clásicas demandas científicas y el ejercicio constante de reflexividad crítica y de auto-reconocimiento propios de una Investigación Acción Feminista» (Francsico Amat et.al, 2015: 155), donde el papel de los/as investigadores/as resulta vital.

1 Departamento de Filosofía y Sociología - Instituto Universitario de Estudios Feministas y de Género Purificación Escribano (Universitat Jaume I); medinam@uji.es

2 Departamento de Filosofía y Sociología - Instituto Universitario de Estudios Feministas y de Género Purificación Escribano (Universitat Jaume I); reverter@uji.es

3 Maria Medina-Vicent y Sonia Reverter-Bañón se encuentran dentro del Proyecto de Investigación Científica y Desarrollo Tecnológico FI2013-47136-C2-2-P, financiado por el Ministerio de Economía y Competitividad del Gobierno de España; y del Proyecto de Investigación Científica y Desarrollo Tecnológico P1.1B2013-24, financiado por el Plan de Promoción de la Investigación de la Universitat Jaume I de Castelló. 
Al mismo tiempo, la lectura de este número nos lleva a subrayar la importancia de incorporar una formación en materia de género dentro de los estudios universitarios (Donoso-Vázquez y Velasco-Martínez, 2013), realidad que desde aquí queremos reivindicar, ya que dicha formación contribuye a la formación democrática de la ciudadanía y por tanto, a la construcción del espíritu crítico tan necesario en nuestros días (Reverter-Bañón, 2001; 2011). Así pues, teniendo en cuenta estas cuestiones y en vistas a elaborar un mapa del número que aquí estamos presentando, pasaremos a comentar brevemente las ideas centrales de las secciones que conforman Asparkía, y que son: articles, retrat, textos, creació literària y llibres.

En primer lugar, encontramos el artículo titulado «La lectora decimonónica de textos intelectuales en su rol como agente social» de Pedro García Suárez (Universidad Internacional de La Rioja, España), donde el autor estudia la manera en que la lectura de carácter intelectual afecta a la construcción de la heroína perteneciente a la novela realista y naturalista española en la relación con su entorno. De este modo, desde el campo de la literatura, García Suárez nos acerca a los procesos identitarios de construcción de la heroína en la novela, que surgen a través de la creación literaria de su interacción con otros personajes.

En segundo lugar, Yolanda Cano Cabrera (Universidad de Granada, España) aborda el concepto de amor predominante en la sociedad occidental y sus efectos en la dependencia emocional de las mujeres con respecto a sus parejas. Su artículo «Nosotras las occidentales, ¿empoderadas emocionalmente? Comentarios a partir de un estudio etnográfico en la ciudad de Granada», nos remite a un trabajo etnográfico desarrollado por la autora en el Barrio del Realejo (Granada), donde se nos muestra que mujeres occidentales supuestamente empoderadas, tienen un conjunto de limitaciones en su autonomía y libertad debido a su dependencia emocional respecto a los hombres.

En tercer lugar encontramos a Julieta Evangelina Cano (Universidad Nacional de La Plata, Argentina), quien reflexiona sobre la construcción patriarcal de la otredad femenina en una posición inferior dentro de la dinámica social, en su trabajo «La "otredad" femenina: construcción cultural patriarcal y resistencias feministas». La autora se centra en el concepto de grupo nominador que construye un signo que señala la inferioridad anclada en la diferencia genital, cimiento de la arquitectura de los cuerpos sexuados, inventando al grupo signado como subalterno. A partir de la lectura crítica de producciones de los estudios culturales, y la utilización de categorías clave como cultura e identidad, se visibilizan las resistencias a la construcción patriarcal de la otredad femenina como subyugada a la masculina.

En cuarto lugar, encontramos el trabajo de Belén León-Río (Universidad de Sevilla, España) titulado «Artistas del siglo XX y XXI en busca del matriarcado perdido». A lo largo de su artículo, León-Río nos introduce en el mundo del arte para estudiar cómo la feminidad ha sido construida en dicho espacio como un poder que se erige contra el racionalismo patriarcal dominante en nuestras sociedades. Desde su perspectiva, a través del arte se puede transformar el lenguaje binario y jerarquizado que separa los significados de lo que supone ser mujer u hombre, significados que pueden reencontrarse en el arte para edificar un término medio positivo que reconcilie lo masculino y lo femenino que se halla en todo ser humano. 
En quinto lugar, Teresa Alzás García (Universidad Isabel I, España), Carmen Galet Macedo (Universidad de Extremadura, España), y Jane Felipe Souza (Universidade Federal do Rio Grande do Sul, Brasil), nos presentan su investigación «Análisis de la deseabilidad social de los roles de género». En este estudio se analiza la deseabilidad social de los sesenta ítems que contiene el Sex Role Inventory de Sandra Bem (BSRI) elaborado en 1974, uno de los instrumentos de medida más utilizados en las investigaciones que analizan los constructos conceptuales relacionados con la identidad de género. El objeto central del análisis es conocer si existen cambios en las atribuciones de los roles de género producidas en los últimos años con respecto al año de definición del Sex Role Inventory.

En sexto lugar, Natalia Lorena Zorrilla Sirlin (Consejo Nacional de Investigaciones Científicas y Técnicas, Argentina) aborda las lecturas e interpretaciones que la polémica obra de Sade ha suscitado en el pensamiento feminista. Su artículo titulado «Sade en el pensamiento feminista» recorre dichas críticas analizando en particular el concepto libertinage sadien y concentrándose en el tratamiento de la diferencia sexual en las novelas libertinas de Sade y sus posibles aportes a la reflexión sobre la igualdad de género en el seno social. Al mismo tiempo, la autora caracteriza la obra de Sade como una vía de defensa de sexualidades alternativas, y un modelo de erotismo sacrificial, cercano a lo que se denomina «saidsmo».

En séptimo lugar, Adriana M. Álvarez Suárez y Priscyll Anctil Avoine (Universidad de Santo Tomás, Colombia) presentan «El feminismo desde lo indígena: trayectorias de estudiantes Wayuu en la Universidad Santo Tomás de Bucaramanga». Mediante este trabajo de investigación, las autoras abordan el encuentro intercultural que se produjo en el año 2009 en la Universidad Santo Tomás de Bucaramanga (Colombia), con la llegada de estudiantes wayuu a dicha institución. A partir del feminismo indígena, la teoría decolonial y el método biográfico, este artículo analiza las trayectorias de vida que definen las subjetividades del estudiantado y que atraviesan sus cosmovisiones mitológicas, culturales, educativas y de género. De este modo evidencian cómo se define lo femenino y lo masculino con relación a los cambios territoriales, epistemológicos, pedagógicos y socioculturales a los cuales se ven enfrentado/as en su nuevo escenario de aprendizaje.

En octavo lugar, Belén Ruíz Garrido (Universidad de Málaga, España) nos introduce de nuevo una cuestión relacionada con el mundo del arte en el trabajo «Mujeres y árboles. Asimilaciones naturales y autorepresentaciones feministas». Ruíz Garrido redefine el papel que juega el árbol en el arte. En este sentido, la presencia del árbol en las producciones artísticas pasaría de erigirse como un referente fálico, a un alter ego fuerte y poderoso, motivo creativo desde posiciones feministas diversas entre las que se inscribe el ecofeminismo. Este trabajo pretende señalar las pautas de la pertinencia de este referente natural en los discursos feministas sobre el cuerpo, el sujeto y la identidad.

En último lugar, Alma López Vale (Universidad Nacional de Educación a Distancia, España), presenta su trabajo «De histérica inválida a escritora transgresora: el caso de Alice James y la superación de los límites». En su aportación, López Vale analiza los resquicios que quedan en nuestra sociedad de la histeria como categoría 
médica. Para desarrollar su trabajo, la autora se centra en el caso de Alice James, quien sufrió las consecuencias de dicha categoría a través de su enfermedad: una invalidez que la postró en cama durante más de la mitad de su vida.

Como se puede observar, el mapa de temáticas y reflexiones que nos presentan los/as autores/as de este número de Asparkía es tan diverso como apasionante. Además de los artículos, la sección Retrat nos invita a conocer la interesante figura de Marjane Satrapi, la directora de cine y autora de cómics iraní-francesa. Una de sus más afamadas obras es «Persépolis», novela gráfica autobiográfica que muestra cómo era la vida en el Irán en el que vivió Marjane, recuperando las voces silenciadas que allí existen aún en la actualidad. A través de su obra, Satrapi se erige como testigo vivo de la realidad histórica iraní, y la traslada al resto del mundo, en un esfuerzo por desmontar viejos estereotipos que la visión occidental del mundo edifica sobre lo iraní. La tolerancia es una valor que impregna la vida y obra de esta autora (Hermoso, 2007), cuya voz representa la de muchas otras que transitan entre dos mundos, que aunque pueden parecer opuestos, tienen mucho en común en lo que se refiere a desigualdad de género.

La sección textos nos ofrece la obra de Clorinda Matto de Turner (1852-1909), escritora peruana y precursora de la novela hispanoamericana e indigenista. Incorporamos en este número su texto «Las obreras del pensamiento en la América del Sur del 1895», concretamente la lectura hecha por la autora en el Ateneo de Buenos Aires el día 14 de diciembre del año 1895. Con la lectura de este texto, Matto de Turner «elabora una cartografía de la formación de la figura de la escritora profesional en América Latina» (Ferrús, 2013: 121), configurándose así como una contribución relevante para la re-construcción del modelo de mujer vigente en el siglo XIX. Al mismo tiempo, su obra es el ejemplo de la lucha contra las dificultades por las que tenían que atravesar las escritoras del momento para cumplir con las exigencias del mundo de la escritura, dominado por los hombres.

Al mismo tiempo, debemos señalar que tanto las ilustraciones del número 29 de Asparkía como la creació literària, se las debemos al trabajo artístico de Sandra Johana Silva Cañaveral y al equipo que colaboró para crear el proyecto «Vestidos Orales». Dicho proyecto se configura como un espacio de encuentro literal y metafórico entre la comunidad de mujeres transgénero que ejercen la prostitución en el Parque La Libertad (Risaralda, Colombia) y la comunidad universitaria de la misma población que recoge a profesionales del arte, estudiantes y docentes. Cruzando fronteras tanto geográficas como identitarias, ambos grupos construyen un recurso que permite la valorización de nuevas identidades de género no solamente durante el tiempo que el proyecto estuvo en activo y en la Marcha por la Diversidad Sexual y de Género del 28 de junio de 2015, sino también como transformación de los imaginarios sociales sobre la diversidad sexual en Colombia. Así pues, esta obra fruto del esfuerzo colectivo supone un paso clave para repensar la homonormativización de nuestras sociedades (López Clavel, 2015) hacia modelos más inclusivos y diversos.

En la sección Llibres, encontramos tres recensiones que nos ofrecen una oportuna actualización sobre las últimas novedades en publicaciones relacionadas con la teoría feminista. En primer lugar, María Victoria Goicoechea Gaona (Universidad 
Nacional del Comahue, Argentina) reseña la obra «Chicas que entienden. In-visibilidad lesbiana» de María Ángeles Goicoechea Gaona, Olaya Fernández Guerrero, María José Clavo Sebastián y Remedios Álvarez Terán (2015). En segundo lugar, María Angélica Cuevas Calderón (Universitat Jaume I) reseña la obra «La AMAEM Marías Guerreras: Asociacionismo de mujeres y acción cultural» de Itziar Pascual Ortiz (2014). Por último, Magdalena Sancho Moreno (Universitat Jaume I) elabora la recensión de «Maternidades subversivas», obra de María Llopis (2014).

En resumen, solamente nos queda dar las gracias a las autoras y los autores que han colaborado en este número de Asparkía, haciéndonos partícipes no sólo de su trabajo, sino de su pasión y su compromiso con la construcción de una sociedad más justa e igualitaria, sea desde el campo de investigación que sea. La edición de «Miradas feministas que interrogan» ha supuesto para nosotras un ejercicio de comprensión sobre la heterogénea realidad de nuestras sociedades y de la existencia de la desigualdad de género en su seno. De esta manera y «con el objetivo prioritario de echar luz tanto sobre la importancia de la interdisciplinaridad como de la introducción de la perspectiva de género en el terreno de la investigación social» (García Pastor, 2012: 104), lanzamos este número de Asparkía para que permita a cada cual interrogar su propio yo y su entorno para transformar críticamente los espacios de injusticia que perviven hoy.

\section{BIBLIOGRAFÍA}

CrenshaW, Kimberle (1989): «Demarginalizing the Intersection of Race and Sex: a Black Feminist Critique of Antidiscrimination Doctrine, Feminist Theory and Antiracist Politics, University of Chicago Legal Forum, Vol. 1989: iss. 1, Article 8, pp. 139-167.

Donoso-Vázquez, Trinidad y Velasco-Martínez, Anna (2013): «¿Por qué una propuesta de formación en perspectiva de género en el ámbito universitario», Profesorado. Revista de currículum y formación del profesorado, Vol.17, Nº1, pp. 71-88.

FERRús, Beatriz (2013): "Las “Obreras del pensamiento" y la novela de folletín (Rosario Orrego de Uribe, Lastenia Larriva de Llona y Josefina Pelliza de Sagasta)», Lectora, $\mathrm{N}^{\circ} 19$, pp. 121-135.

Francisco Amat, Andrea, Lozano Estivalis, María y Traver Martí, Joan (2015): «Paradojas epistemológicas de una investigación participativa feminista», Asparkía. Investigació Feminista, No26, pp. 155-169.

García PAstor, Begoña (2012): «La perspectiva de género en el ámbito etnográfico y socioantropológico», en TORRENT EsClapÉs, Rosalía y REvERTER-BAÑón, Sonia (Ed.): Variaciones sobre género, pp. 103-112.

HERMOSO, Borja (2007): «El odio viaja en nuestra genética», Disponible en: http:/ / elpais.com/diario/2007/10/26/cine/1193349601_850215.html [Consultado el 11 de noviembre de 2016] 
López Clavel, Pau (2015): «Tres debates sobre la homonormativización de las identidades gay y lesbiana», Asparkía. Investigació Feminista, No26, pp. 137-153.

LóPEZ SAAVEDRA, Lisbett (2011): «La teoría de género y sus heterogéneas perspectivas, un reclamo pertinente», en Contribuciones a las Ciencias Sociales, Disponible en www.eumed.net/rev/cccss/15/ [Consultado el 11 de noviembre de 2016]

REVERTER-BAÑón, Sonia (2001): «Feminismo y democracia: una crítica antifundamentalista», Recerca. Revista de Pensament i Anàlisi, No1, pp. 95-108.

- (2011): «La dialéctica feminista de la ciudadanía», Athenea Digital, Vol.11, No3, pp. 121-136.

Silva CaÑAveral, Sandra Johana (2016): «Vestidos orales», Disponible en: https:/ / www.youtube.com/watch?v=10bYEwrkjh8 [Consultado el 11 de noviembre de 2016]

Verloo, Mieke (2005): «Mainstreaming Gender Equality in Europe. A Critical Frame Analysis Approach», The Greek Review of Social Research, N¹17, pp. 11-34. 